\title{
Uso do Alongamento Estático Como Fator Interveniente na dor Muscular de Início Tardio
}

\section{Use of Static Stretching as an Intervenient Factor in Delayed Onset Muscle Soreness}

Aline Evans de Oliveira Bonfim Daniela De Rél

Juliana Gaffuri'

Marcela Medeiros de Almeida Costa' José Luiz Marinho Portolez ${ }^{2}$

Gladson Ricardo Flor Bertolini'

1. Universidade Estadual do Oeste do Paraná - Unioeste - Laboratório de Estudo das Lesões e Recursos Fisioterapêuticos - Cascavel, PR. 2. Universidade Santa Cecília Unisanta - Santos, SP.

Endereço para correspondência: Gladson Ricardo Flor Bertolini Rua Universitária, 2.069, Jd. Universitário, Caixa Postal: 711 85819-110 - Cascavel, PR E-mail: gladsonricardo@gmail.com

\begin{abstract}
RESUMO
Introdução: A dor muscular de início tardio consiste em uma sensação de desconforto muscular consequente da prática de exercício físico intenso, que perdura durante alguns dias. O alongamento estático pode ser usado para tentar amenizar esse efeito pós-exercício, mantendo-o durante cerca de 10-30 segundos e repetindo o procedimento por três a cinco vezes. Objetivo: Verificar, em indivíduos sedentários, o efeito do alongamento estático para o alívio da dor muscular de início tardio. Métodos: Este estudo foi um ensaio clínico randomizado, prospectivo, cego por parte do avaliador, composto por 20 estudantes que foram divididos em dois grupos: GAL (exercício + alongamento) e GC (exercício). O exercício foi constituído de cinco séries com 20 repetições de planti/dorsiflexão, exercitando o grupo tríceps sural. Ambos os grupos foram avaliados antes do exercício e reavaliados após 24, 48 e 72 horas, quanto ao seu grau de dor utilizando-se a escala visual analógica (VAS) e um dolorímetro de pressão. Resultados: A VAS mostrou que no grupo controle (GC) houve diferença significativa na sensação de dor no pré-exercício comparado a 24, 48 e 72 horas, entre 24 e 72 horas e entre 48 e 72 horas após o exercício. No grupo alongamento (GAL), a VAS mostrou diferenças significativas no período pré-exercício comparado com 24, 48 e 72 horas e entre 48 e 72 horas após o exercício. O dolorímetro mostrou que no grupo controle (GC), houve diferença significativa na sensação de dor no pré-exercício comparado a 24 e 48 horas e entre 24 e 72 horas após o exercício. No grupo alongamento (GAL), as diferenças significativas se mostraram no período pré-exercício comparado com 24 e 48 horas após o mesmo. Conclusão: O alongamento estático não foi eficaz para o alívio da dor muscular de início tardio no grupo avaliado.
\end{abstract}

Palavras-chave: músculo esquelético, flexibilidade, medida de dor.

\begin{abstract}
Introduction: The Delayed Onset Muscle Soreness consists in a sensation of muscle discomfort resulting from the practice of intense physical exercise that lasts for some days. Static stretching can be used to try to diminish this post-exercise effect, holding it for about 10-30 seconds and repeating the procedure three to five times. Objective: To verify, in sedentary individuals, the effect of the static stretching to relieve the Delayed Onset Muscle Soreness. Methods: This study was a randomized, prospective, blind by the evaluator clinical trial, composed of 20 students divided in two groups: STG (exercise + stretching) and CG (exercise). The exercise was composed of five sets of 20 repetitions of plantar/dorsiflexion, exercising the triceps sural group. Both groups were evaluated before exercising and again after 24,48 and 72 hours, as to their pain degree using the Visual Analog Scale (VAS) and a Dolorimeter pressure. Results: The VAS showed that control group (CG) presented significant difference in pain sensation in the pre-exercise compared to 24,48 and 72 hours, between 24 and 72 hours and between 48 and 72 hours after exercising. Stretching group (STG) VAS showed significant differences in the pre exercise period compared to 24,48 and 72 hours and between 48 and 72 hours after exercising. The dolorimeter showed that in control group (CG) there was significant difference in pain sensation in pre exercise compared to 24 and 48 hours and between 24 and 72 hours after exercising. In stretching group (STG), the significant differences were evident in the pre exercise period compared to 24 and 48 hours after it. Conclusion: Static stretching was not effective in relieving Delayed Onset Muscle Soreness.
\end{abstract}

Keywords: skeletal muscle, flexibility, pain measurement. 


\section{INTRODUÇÃO}

A dor é um mecanismo de proteção do organismo, contra uma agressão tecidual, e pode ser desencadeada por vários tipos de agentes, que estimulam terminações nervosas livres presentes em toda a superfície corporal (nociceptores). Ela é caracterizada por uma experiência sensorial e emocional, de caráter desagradável, associada à injúria tecidual(1).

Neste contexto, as dores musculares e a sensação de desconforto consequentes da prática de atividade física, intensa, constituem um dos quadros mais comumente encontrados, tanto em atletas profissionais como em atletas de final de semana ${ }^{(2)}$. Até mesmo indivíduos sedentários já experimentaram alguma vez na vida um episódio de dor muscular, principalmente após a execução de um padrão de movimento diferente daquele ao qual estão acostumados ${ }^{(3)}$.

A dor transitória sentida por estes indivíduos é denominada dor muscular de início tardio (DMIT) ${ }^{(2)}$. A DMIT é uma sensação de desconforto na musculatura esquelética caracterizada por dor, edema, perda da amplitude de movimento com consequente diminuição da flexibilidade, perda de força muscular do membro afetado, aumento dos níveis séricos de creatina cinase, desidrogenase láctica, mioglobina e fragmentos da cadeia pesada de miosina. Ainda, a manifestação subjetiva de dor muscular é consequência de microtraumas nas fibras musculares, que dão início a um processo inflamatório localizado na musculatura solicitada, com manifestações dolorosas intensas que são exacerbadas pela movimentação ou palpação(4). A DMIT geralmente ocorre oito horas após o término do exercício, alcança intensidade máxima entre 24 e 72 horas e desaparece entre cinco e sete dias ${ }^{(5,6)}$.

A origem desse desconforto muscular verificado após a realização do exercício físico pode estar relacionada com fenômenos de natureza metabólica e/ou com aumento da resistência periférica, que gera alteração do fluxo sanguíneo nos grupos musculares exercitados e aumento no perímetro do membro(7).

Para amenizar tais dores sugere-se a realização do alongamento após a prática de esportes ou após competição ${ }^{(7)}$. 0 alongamento consiste em qualquer forma de trabalho submáximo, que visa a manutenção da flexibilidade e a realização de movimentos de amplitude normal com o mínimo de restrição física possível|(8).

A aplicação do alongamento tem a finalidade de prevenir ou diminuir os efeitos agressivos do exercício, aumentar a recuperação muscular pela capacidade de dispersar o edema, aumentar a amplitude articular e reduzir espasmos musculares. Usado, também, como tentativa de melhorar o desempenho muscular e desta forma, reduzir os riscos de lesão. Ao realizar alongamentos repetidos e sustentados, em qualquer extensão, há redução de tensão na unidade tendínea muscular e alterações no limiar sensitivo dos receptores de dor $r^{(6,7,9,10)}$.

Diversos são os métodos e técnicas com objetivo de promover o alongamento, como o tipo estático, balístico e a técnica de facilitação neuromuscular proprioceptiva (FNP) ${ }^{(11)}$.

O alongamento estático caracteriza-se por movimentos lentos, até o ponto máximo de amplitude articular ou até o ponto de desconforto do indivíduo, mantendo-o, em geral, durante cerca de 10-30 segundos. Para que se obtenha efeito ótimo, o alongamento estático deve ser realizado sequencialmente de três a cinco vezes ${ }^{(12)}$.

Portanto, visto a possibilidade de alteração do limiar de dor pela realização do alongamento estático, pós-exercício, este poderia ser fator favorável na redução da dor muscular de início tardio(11). Atualmente, vários benefícios do alongamento são conhecidos. Porém, há poucos estudos que relacionam seu efeito com a dor muscular de início tardio. O objetivo deste estudo foi verificar, em indivíduos sedentários, o efeito do alongamento estático para o alívio da dor muscular de início tardio de tríceps sural.

\section{MÉTODOS}

\section{Caracterização do estudo e amostra}

Este estudo é caracterizado como ensaio clínico randomizado, transversal, cego por parte do avaliador. O estudo foi realizado no Laboratório de Lesões e Recursos Fisioterapêuticos da Universidade Estadual do Oeste do Paraná, campus Cascavel, com uma amostra de conveniência composta por 20 indivíduos de ambos os sexos, estudantes da instituição. A amostra foi dividida em dois grupos: Grupo Controle ou GC $(n=10)$ que realizou somente exercício e Grupo Alongamento ou GAL ( $n=10$ ) que realizou exercício e alongamento. Ambos foram avaliados em relação à dor pós-exercício.

Os critérios de inclusão foram: faixa etária entre 17-30 anos e sedentarismo. Como critérios de não inclusão: possuir algum tipo de doença que contraindicasse a prática de exercício ou de alongamento e não se enquadrar nos critérios de inclusão.

O trabalho foi aprovado pelo Comitê de Ética em Pesquisa da Universidade Estadual do Oeste do Paraná, sob protocolo no 266/2009-CEP.

\section{Protocolo de exercício e alongamento}

O exercício proposto foi de plantiflexão e dorsiflexão, realizado com o individuo em posição ortostática, realizando contração concêntrica e excêntrica, respectivamente, do grupo tríceps sural. Para realizar o exercício o indivíduo apoiava-se no corrimão de uma escada, com os antepés sobre o degrau, sendo o próprio peso corporal a carga utilizada.

Esse procedimento foi realizado com os dois grupos, apenas no primeiro dia do estudo, e consistiu em cinco séries de 20 repetições, com intervalos de 30 segundos entre as séries. Antes da realização do exercício foi verificada a pressão arterial de cada indivíduo, apenas para controle.

O grupo GC foi submetido à avaliação e exercício. As etapas do estudo para o grupo GC consistiram em:

- 10 dia - avaliação e exercício de planti/dorsiflexão;

- $2^{\circ}$ dia (24 horas pós-exercício) - reavaliação;

- 30 dia (48 horas pós-exercício) - reavaliação;

- $4^{\circ}$ dia (72 horas pós-exercício) - reavaliação.

O grupo GAL fez a avaliação e a sessão de exercício e, logo após, o alongamento estático, de forma ativa, da musculatura posterior da perna (tríceps sural). O alongamento foi realizado com o individuo em pé, inclinado na direção de uma parede, com os pés apoiados no chão, um à frente do outro, e o joelho da perna anterior ligeiramente fletido. Os indivíduos foram instruídos a inclinarem-se lentamente para frente, aumentando a flexão do joelho, mantendo o calcanhar da perna posterior apoiado no chão, até ser percebido um estiramento do músculo tríceps sural. Esta posição foi mantida durante 30 segundos e, após, realizou-se o mesmo procedimento com o membro contralateral. 0 alongamento foi repetido três vezes em cada perna.

As etapas do estudo para o grupo GAL consistiram em:

- 10 dia - avaliação, exercício de plantiflexão/dorsiflexão e alongamento;

- $2^{\circ}$ dia (24 horas pós-exercício) - reavaliação e alongamento;

- $3^{\circ}$ dia (48 horas pós-exercício) - reavaliação e alongamento;

- $4^{\circ}$ dia (72 horas pós-exercício) - somente reavaliação.

\section{Avaliação da dor}

A avaliação da dor foi realizada pelos seguintes instrumentos: escala visual analógica (VAS) e dolorímetro de pressão. A VAS consiste em uma escala numérica de 0-10, onde zero significa ausência total de dor e 10 refere-se à dor máxima suportada pelo indivíduo. O voluntário foi questionado a respeito de sua dor e marcou o grau desta, na escala.

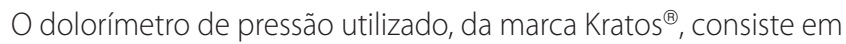
um aparelho capaz de exercer uma pressão de até 50kgf (quilogramas- 
força). A pressão foi efetuada no ventre muscular do músculo gastrocnêmio medial (entre o primeiro e o segundo terço do músculo referido). Inicialmente, essa pressão foi mínima, aumentando até o ponto em que o paciente referiu dor.

\section{Análise estatística}

Os dados coletados foram analisados estatisticamente pelos seguintes métodos: teste de D'Agostino \& Pearson para avaliar a normalidade dos dados, Análise de Variância (ANOVA) medidas repetidas para análise intragrupos e teste $t$ de Student não pareado para análise intergrupos. O nível de significância aceito foi 5\%.

\section{RESULTADOS}

Ao comparar as amostras, elas foram consideradas homogêneas pelo teste $t$ de Student não pareado, o qual manteve-se constante em todos os momentos avaliados, tanto pela VAS quanto pelo dolorímetro de pressão $(p>0,05)$.

Ao confrontar os resultados intragrupos, pela escala visual analógica (VAS), verificou-se que no grupo controle (GC) houve diferença significativa na sensação de dor no pré-exercício comparado a 24, 48 e 72 horas após o mesmo. Verificou-se também diferença significativa na dor entre 24 e 72 horas, assim como, entre 48 e 72 horas após o exercício (figura 1).

Entre os indivíduos do grupo alongamento (GAL), a VAS mostrou diferenças significativas quanto à dor no período pré-exercício comparado com 24, 48 e 72 horas após o mesmo. A mesma relação foi verificada também entre 48 e 72 horas (figura 1).

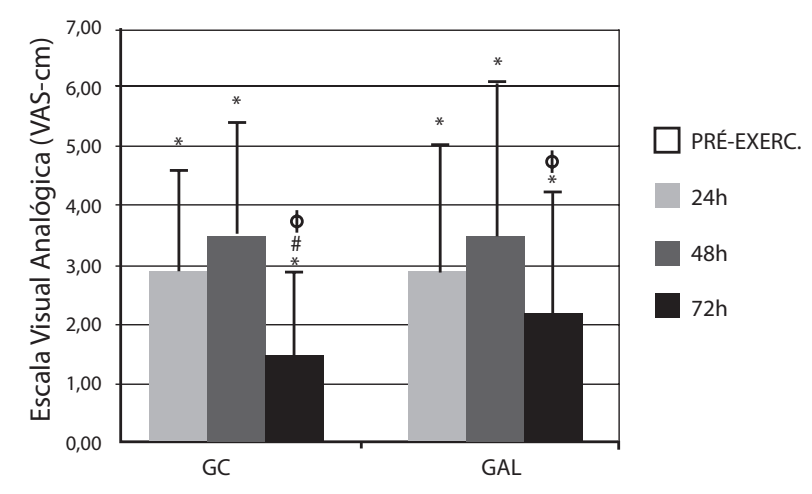

Figura 1. Nível de dor muscular de início tardio avaliado pela escala visual analógica para os grupos controle e alongamento. * Diferença significativa em relação ao pré-exercicio. \# Diferença significativa em relação a 24h pós-exercício. Ф Diferença significativa em relação a 48 h pós-exercício.

A avaliação pelo dolorímetro mostrou que no grupo controle (GC) houve diferença significativa na sensação de dor no pré-exercício comparado a 24 e 48 horas após o mesmo, e entre 24 e 72 horas (figura 2).

Entre os indivíduos do grupo alongamento (GAL), as diferenças significativas mostraram-se no período pré-exercício comparado com 24 e 48 horas após o mesmo (figura 2).

\section{DISCUSSÃO}

Um dos benefícios citados da realização do alongamento estático é que ele requer menos energia e alivia as dores musculares ${ }^{(11)}$. Além disso, com esse tipo de alongamento, obtém-se aumento da flexibilidade e relaxamento, quando realizado de forma isolada ou de maneira global(13). Desta forma, no presente estudo, utilizou-se o alongamento estático visando diminuir a dor muscular de início tardio, comparando a um grupo controle, o qual apenas executou a atividade muscular que originou a dor.

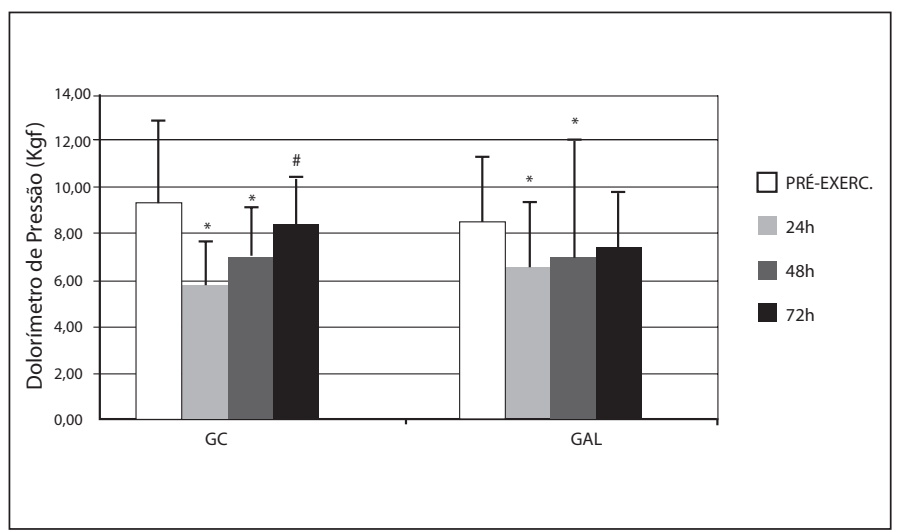

Figura 2. Nível de dor muscular de início tardio avaliado pelo dolorímetro de pressão para os grupos controle e alongamento. * Diferença significativa em relação ao préexercício. \# Diferença significativa em relação a 24 h pós-exercício.

Não há um consenso de como executar o alongamento, de forma a garantir sua máxima eficiência. Isso se deve às dúvidas geradas a partir da diversidade de técnicas, número de repetições, frequência, tempo de duração e sobre a intensidade de tensão que deve ser aplicada ao músculo durante o alongamento. Sugere-se que esta intensidade deveria ser aplicada até o sujeito referir um "incômodo", "desconforto", "tensão sem dor","leve sensação de alongamento"(14). No estudo realizado, aplicou-se o alongamento estático de forma ativa, sendo explicado ao participante que o mesmo deveria sentir uma leve sensação de alongamento, sem a produção de desconforto no músculo alongado.

Os resultados obtidos no estudo mostraram-se desfavoráveis ao uso do alongamento estático ativo, com o intuito de diminuir a dor muscular de início tardio, pois, ao comparar os momentos 24 horas com 72 horas após o exercício, houve redução significativa da dor, tanto pela VAS quanto pelo dolorímetro de pressão, para o grupo controle, fato não observado no grupo alongado. Tal resultado vai de encontro ao estudo realizado por Buroker e Schwane ${ }^{(15)}$, no qual demonstraram que em 23 indivíduos, após a realização de exercício em step, durante 30 minutos, não houve alívio da dor muscular de início tardio imediatamente após o alongamento, nem mesmo três dias após o período de exercício.

High e Howley ${ }^{(16)}$ submeteram voluntários a: a) alongamento estático antes do exercício em step; b) aquecimento antes do exercício em step; c) alongamento estático e aquecimento antes do exercício; e d) somente exercício. $O$ exercício em step foi realizado até a exaustão do voluntário e, após avaliação em 24 horas e cinco dias, verificou-se que o alongamento estático e o aquecimento não preveniram a dor muscular de início tardio. O tipo de movimento e de alongamento utilizado por High e Howley(16) é semelhante ao realizado no presente estudo, porém, o alongamento proposto pelos autores foi executado antes do exercício. Ainda assim, o objetivo de prevenir a dor muscular não foi atingido.

Johansson et al. ${ }^{(17)}$ investigaram os efeitos do alongamento estático pré-exercício na manifestação da dor muscular tardia, estudando 10 mulheres que tiveram esta dor induzida no músculo flexor do joelho em ambas as pernas, com exercício excêntrico seguido de exercício em cicloergômetro. Os resultados do estudo sugeriram que este tipo de alongamento não tem qualquer efeito preventivo na manifestação da dor muscular tardia. De forma concordante, a revisão realizada por Andersen ${ }^{(18)}$ constatou que o alongamento pré ou pós-exercício não é capaz de intervir no alívio da dor muscular.

Wessel e Wan ${ }^{(19)}$ investigaram o uso do alongamento na melhora da dor muscular de início tardio induzida por exercício físico excêntrico e concêntrico e concluíram que o alongamento, realizado antes ou após o exercício físico, não reduziu a dor. Conclusão semelhante 
pode ser vista na revisão bibliográfica de Herbert e Gabriel(20), na qual o alongamento antes ou depois do exercício não protegeu o músculo contra a dor de início tardio. Ao relacionar tais estudos com o atual, pode-se sugerir que o momento de aplicação do alongamento não influencia a diminuição da dor. Portanto, no presente estudo, o uso do alongamento somente após o exercício, pode não ser um fator de interferência nos resultados encontrados.

Em divergência com os estudos apresentados, Laroche et al. ${ }^{(9)}$ submeteram 29 mulheres a alongamento estático e balístico após exercício excêntrico. O objetivo foi avaliar o efeito de quatro semanas destes sobre as propriedades musculares, redução de dano muscular e de dor muscular de início tardio. Os autores verificaram que todos os grupos apresentaram um aumento na dor em 24, 48 e 72 horas após o exercício excêntrico, com pico em 48 horas. Embora não estatisticamente significativo, o grupo controle apresentou dor maior um, dois e três dias após a atividade. Estes dados parecem indicar atenuação na dor muscular nos grupos que realizaram alongamento, o que poderia ser significativo se houvesse um maior número de indivíduos na amostra e um indicador mais sensível de dor. No presente estudo, com maior amostra e aplicação de indicador (dolorímetro de pressão) mais sensível, a dor comportou-se de maneira semelhante, porém a atenuação da dor muscular não foi observada no grupo de alongamento.

Após a análise dos resultados, apresentam-se como limitações a subjetividade dos instrumentos de avaliação e/ou a escolha do alongamento e sua forma de realização. Sugere-se que mais estudos sejam realizados para a elucidação dos resultados obtidos.

\section{CONCLUSÃO}

Com este estudo conclui-se que o alongamento estático não foi eficaz para o alívio da dor muscular de início tardio, levando a níveis de dor ainda mais elevados no grupo alongamento que no grupo controle.

Todos os autores declararam não haver qualquer potencial conflito de interesses referente a este artigo.

\section{REFERÊNCIAS}

1. Lana AC, Paulino CA, Gonçalves ID. Influência dos exercícios físicos de baixa e alta intensidade sobre o limiar de hipernocicepção e outros parâmetros em ratos. Rev Bras Med Esporte 2006;12:248-54

2. Ghorayeb N, Dioguardi GS, Daher DJ, Jardim CA, Baptista CA, Batlouni M. Avaliação cardiológica préparticipação do atleta. Rev Soc Cardiol Estado de São Paulo 2005;15:97-104.

3. Molina R, Amorim AR. Efeito do dano muscular através de diferentes tipos de exercício excêntrico sobre a economia de movimento. RENSC 2007;2:89-99.

4. Barros TL, Angeli G, Barros LFL. Preparação do atleta de esportes competitivos. Revista de Sociologia e Cardiologia do Estado de São Paulo 2005;15:114-20.

5. Tricoli V. Mecanismos envolvidos na etiologia da dor muscular tardia. Rev Bras Ciên e Mov 2001;9:39-44

6. Cheung K, Hume PA, Maxwell L. Delayed onset muscle soreness: treatment strategies and performance factors. Sports Med 2003;33:145-64

7. Torres R, Carvalho P, Duarte JA. Influência da aplicação de um programa de estiramentos estáticos, após contrações excêntricas, nas manifestações clínicas e bioquímicas de lesão muscular esquelética. Rev Port Cien Desp 2005;5:274-87.

8. Galdino LAS, Nogueira CJ, César EP, Fortes MEP, Perrout JR, Dantas EHM. Comparação entre níveis de força explosiva de membros inferiores antes e após flexionamento passivo. Fit Perf J 2005;4:11-5.

9. Laroche DP, Connolly DAJ. Effects of stretching on passive muscle tension and response to eccentric exercise. Am J Sports Med 2006;34:1000-7.

10. Nordez A, Cornu C, Mcnair P. Acute effects of static stretching on passive stiffness of the hamstring muscles calculated using different mathematical models. Clin Biomech 2006;21:755-60.

11. Bandy WD, Iron JM. The effect of time on static stretch on the flexibility of the hamstring muscles. Phys Ther 1994;74:845-50
12. Tirlonil AT, Belchior ACG, Carvalho PTC, Reis FA. Efeito de diferentes tempos de alongamento na flexibilidade da musculatura posterior da coxa. Fisioter Pesq 2008;15:47-52.

13. Morenol MA, Catai AM, Teodori RM, Borges BLA, Cesar MC, Silva E. Efeito de um programa de alongamento muscular pelo método de Reeducação Postural Global sobre a força muscular respiratória e a mobilidade toracoabdominal de homens jovens sedentários. J Bras Pneumol 2007:33:679-86

14. Branco VR, Negrão Filho RF, Padovani CR, Azevedo FM, Alves NE, Carvalho AC. Relação entre a tensão aplicada e a sensação de desconforto nos músculos isquiotibiais durante o alongamento. Rev Bras Fisioter 2006:10:465-72

15. Buroker KC, Schwane JA. Does post exercise static stretching alleviate delayed muscle soreness? Phys Sportsmed 1989;17:65-83

16. High DM, Howley ET. The effect of static stretching and warm-up on prevention of delayed onset muscle soreness. Res Q 1989;60:357-61.

17. Johansson PH, Lindström L, Sundelin G, Lindström B. The effects of preexercise stretching on muscular soreness, tenderness and force loss following heavy eccentric exercise. Scand J Med Sci Sports 1999;9:21925.

18. Andersen JC. Stretching Before and After Exercise: Effect on Muscle Soreness and Injury Risk. J Athl Train 2005;40:218-20

19. Wessel J, Wan A. Effect of stretching on the intensity of delayed-onset muscle soreness. Clin J Sport Med 1994;4:3-7.

20. Herbert RD, Gabriel M. Effects of stretching before and after exercising on muscle soreness and risk of injury: systematic review. BMJ 2002:325:468-73. 